\section{Améliorer la qualité des soins infirmiers en oncologie: modèle de réflexion sur la pratique proposé par les infirmières en oncologie d'Interlink œuvrant en santé communautaire}

\author{
Par Doris Howell et Beth Pelton
}

\begin{abstract}
Abrégé
C'est depuis 1996 que les infirmières en oncologie d'Interlink cuvrant en santé communautaire utilisent, en équipe, la réflexion sur la pratique afin de partager connaissances et expériences entre pairs. La méthode de la réflexion sur la pratique permet à l'infirmière d'examiner la prise de décisions concernant des cas réels et de découvrir les connaissances et l'art qui sous-tendent la pratique infirmière. Cet article décrit l'usage de la réflexion que font des infirmières spécialisées en cancérologie afin d'améliorer la qualité des soins qu'elles prodiguent. L'utilisation d'un cadre structuré pour la réflexion incorporant divers modes d'acquisition du savoir constitue une caractéristique essentielle du modèle Interlink pour la réflexion. L'élaboration d'une démarche visant à guider la réflexion au sein du programme Interlink n'a pas toujours été facile. Toutefois, on estime que l'expérience des infirmières Interlink en matière de réflexion a joué un rôle critique dans le développement continu du programme et dans l'épanouissement des infirmières individuelles. Les infirmières Interlink ont remarqué que la réflexion dirigée ainsi que la création d'un milieu favorable à la réflexion, à la connaissance de soi et à l'autoévaluation sont des éléments fondamentaux du cheminement de l'infirmière privilégiant la réflexion sur la pratique.
\end{abstract}

La réflexion sur la pratique a été décrite comme une méthode permettant de comprendre l'art de la pratique infirmière et de réaliser le plein potentiel thérapeutique (Antrobus, 1997), et de combler le fossé, dans les soins infirmiers, entre la théorie, la pratique et la prise de décision fondée sur des données probantes (Johns, 1996). Les infirmières en oncologie d'Interlink œuvrant en santé communautaire ont utilisé l'auto-réflexion afin d'explorer le vécu de la maladie chez leurs patients atteints de cancer, pour dégager les connaissances qui orientent la prise de décision et la pratique et pour éclairer les interventions infirmières utilisées tout au long du processus thérapeutique. La démarche de réflexion aide l'infirmière Interlink à dégager les raisons pour lesquelles on adopte telles ou telles manières de soigner les patients et à explorer de nouvelles avenues pour ce qui est du soutien offert aux patients tout au long de l'expérience du cancer.

Les infirmières en oncologie d'Interlink forment un organisme de soins infirmiers communautaires à but non lucratif constitué sur le modèle du programme des infirmières Macmillan au Royaume-Uni
(Bunn, 1988). Les infirmières Interlink œuvrent à titre de consultantes indépendantes qui prodiguent des soins infirmiers en oncologie spécialisés à des patients et à leurs proches tout au long du continuum du cancer, depuis le diagnostic précoce jusqu'à la guérison ou la rémission ou jusqu'à la palliation et le soutien aux endeuillés. Les infirmières Interlink participent à la gestion clinique des symptômes de la maladie et des effets secondaires des traitements tout en fournissant un soutien psychosocial essentiel. Elles habilitent les adultes et les enfants atteints de cancer et leurs proches à accéder aux soins et au soutien dont ils ont besoin à chacune des phases de la maladie. Elles se donnent pour buts d'alléger le fardeau que représente le vécu du cancer, de promouvoir un fonctionnement et un bien-être optimaux et d'améliorer la qualité de vie - ou pour le moins de la maintenir à son niveau existant. Les infirmières Interlink se font également personnes-ressources spécialisées auprès des professionnels de la santé qui œuvrent à leurs cotés pour répondre aux besoins des personnes aux prises avec le cancer.

La nature autonome de la pratique de l'infirmière Interlink et la prestation de consultations de soutien relatives aux autres professionnels de la santé communautaire exigent des infirmières Interlink qu'elles fassent constamment progresser leur expertise si elles veulent obtenir des résultats positifs pour leurs patients et montrer la valeur de leur contribution aux soins des personnes atteintes de cancer et de leurs proches. Les infirmières Interlink font appel à la réflexion pour approfondir leur compréhension des assises empiriques et humanistes de la prestation des soins et pour améliorer la qualité des soins qu'elles dispensent.

La réflexion sur les soins prodigués peut permettre aux infirmières d'acquérir une connaissance approfondie de leur pratique et peut constituer une occasion d'apprentissage. L'utilisation d'une démarche fondée sur la réflexion en vue d'explorer les soins infirmiers prodigués aux personnes atteintes de cancer peut fournir de riches détails sur leur vécu de la maladie et sur le rôle thérapeutique de l'infirmière. Les infirmières Interlink ont rapporté que la démarche de réflexion stimule leur épanouissement et leur développement personnels, donne un sens plus profond à leur pratique individuelle, rehausse les relations avec les patients et améliore la résolution de problèmes. On estime que l'utilisation de la réflexion dans la pratique contribue à améliorer l'assimilation de connaissances profondes (Lowe et Kerr, 1998) et à accroître l'efficacité de la résolution de problèmes par le biais d'une démarche d'autoévaluation (Kolb, 1975).

La réflexion sur la pratique aide également les infirmières Interlink à déterminer les lacunes au niveau de leurs connaissances et les sujets qu'elles pourraient couvrir dans leur apprentissage futur. Par exemple, en se penchant sur la réaction d'un adolescent face au cancer, les infirmières Interlink ont décidé qu'elles devaient étudier en profondeur les étapes de la croissance et du développement humains ainsi que les domaines de l'adaptation et du deuil chez les adolescents. La réflexion sur la pratique constitue une méthode en vertu de laquelle les infirmières peuvent évaluer leur propre niveau de compétence en vue de conserver leur immatriculation en Ontario (Wansbrough, 1996). Schon (1983) et Powell (1989) pensent que l'aptitude à la réflexion est une composante essentielle de la compétence en soins infirmiers, qu'elle engendre des praticiennes d'une grande efficacité et qu'elle appuie le perfectionnement de ces dernières.

\section{Modèle conceptuel pour la réflexion}

Au sein du programme Interlink, on a dégagé les facteurs critiques de succès suivants pour la mise en œuvre de la réflexion sur la pratique: utilisation d'un modèle conceptuel pour orienter la réflexion, développement de la connaissance de soi ou de l'auto-

Doris Howell, inf, MScN, $\mathrm{PhD}(\mathrm{c})$, Recherche et Évaluation, Interlink Community Cancer Nurses et conseillère indépendante en soins oncologiques/palliatifs, Lisle, Ontario.

Beth Pelton, inf, BScN, CON(C), Consultante en soins infirmiers, Interlink Community Cancer Nurses, Toronto, Ontario. 
réflexion, création d'un environnement propice à la réflexion.

Johns (1995) a indiqué qu'il fallait commencer par un modèle qui fournirait l'orientation et la structure nécessaires et faciliterait le développement des compétences relatives à la réflexion. La mise en œuvre d'un modèle structuré pour la réflexion aide les infirmières Interlink à effectuer l'analyse critique d'un cas plutôt que de se contenter de rapporter l'histoire du patient et des événements entourant l'interaction de l'infirmière avec ce dernier. Un modèle structuré pour la réflexion permet à la praticienne de saisir dans les situations des significations plus profondes, de révéler les connaissances théoriques et scientifiques utilisées et d'explorer avec ses pairs de nouvelles connaissances ou expériences qui auraient pu être utiles sur le plan des soins au patient. Le modèle de réflexion structurée proposé par Johns (1995), qui incorpore les modes d'acquisition des connaissances de Carper (1978), a été adapté comme cadre d'orientation de la démarche de réflexion au sein du programme Interlink (voir le tableau un).

Ce cadre est extrêmement utile parce qu'il donne à l'infirmière la possibilité d'explorer non seulement la base scientifique ou les données probantes intégrées aux soins infirmiers mais aussi l'art de la prestation de soins. Il a été dit des quatre schémas d'acquisition du savoir définis par Carper qu'ils constituent la voie grâce à laquelle on peut saisir la totalité de la situation de soins infirmiers (Boykin et Schoenhofer, 1991). Des cas critiques sont décrits et explorés à la lumière des quatre paramètres de l'acquisition du savoir infirmier: dimension empirique, dimension morale (éthique), dimension personnelle et dimension esthétique (Carper, 1978). Le cadre fournit une structure propice à la réflexion sur les soins; il guide l'infirmière dans sa découverte des connaissances qui sous-tendent sa pratique et les connaissances additionnelles qui ont pu y être incorporées afin de rehausser les soins infirmiers thérapeutiques. Le modèle favorise la capacité de l'infirmière à réfléchir sur son propre comportement de pratique en explorant celui des paramètres de l'acquisition des connaissances qui a influé sur l'épisode de soin et a entraîné l'adoption d'interventions infirmières et d'une prise de décisions particulières.

\section{Élargissement du cadre de réflexion}

Les infirmières Interlink font souvent face à des situations de soins qui les amènent à négocier et à défendre les intérêts des patients en vue d'obtenir les ressources permettant de répondre aux besoins des patients atteints de cancer et de leurs proches. Ainsi, la prise de conscience de l'environnement social et politique de la prestation des soins devient un élément important du rôle des infirmières Interlink. Ceci les amène à explorer les manières dont elles peuvent utiliser, dans leur pratique, les principes de la promotion de la santé communautaire et de la défense des intérêts des patients. Grâce à la démarche de réflexion, les infirmières Interlink dégagent les enjeux relatifs au système de soins qui exigent des changements au niveau du système ainsi que des actions politiques. Par exemple, une infirmière Interlink a écrit une lettre au président du comité consultatif communautaire d'un centre anticancéreux en vue d'y donner une voix aux personnes recevant des soins dans un système restructuré des soins aux personnes atteintes du cancer. Dans un autre cas, une infirmière Interlink et une étudiante infirmière ont rencontré les femmes d'un ensemble immobilier afin de les informer en matière de santé du sein, elles qui utilisent rarement les programmes structurés de dépistage du cancer du sein. Par conséquent, on a ajouté au cadre de réflexion un cinquième mode d'acquisition des connaissances, le savoir sociopolitique (White, 1995).

Le savoir sociopolitique a été jugé utile à la fois pour l'activisme en faveur du changement dans la collectivité (Hagedorn, 1995) et pour la compréhension des rapports de force qui peuvent influer sur la santé au sein d'une collectivité (Sheilds et Lindsey, 1998). Les infirmières Interlink apprennent, par le biais de la réflexion, à se sensibiliser aux diverses manières d'influencer le changement afin de mobiliser un réseau de soutien autour de la personne atteinte de cancer et de ses proches. Les infirmières Interlink examinent le besoin qu'elles ont de militer au niveau politique à la lumière de leurs ressources personnelles, de leur énergie et de leur propre système de croyance.

Lorsque les infirmières Interlink explorent les situations de soins, elles mettent fréquemment au jour l'art de dispenser des soins ou un élément de nature intuitive (Benner, 1984) qui orientait la pratique infirmière. Selon Johns (1995), le mode esthétique d'acquisition des connaissances est au centre du savoir relié à la pratique, et il est éclairé par les dimensions empirique, personnelle et éthique de la pratique. Il a été établi que les réponses d'un patient individuel et de ses proches à un diagnostic de cancer ne peuvent pas toujours être décrites selon une perspective théorique ou scientifique. Les réponses et les réactions des patients au vécu du cancer sont souvent complexes et se rapportent à la signification que la personne attribue à son vécu et à son expérience du cancer (Howell, 1998). Ces situations ne peuvent être comprises qu'en écoutant l'histoire du patient et en en saisissant la portée réelle. La relation entre l'infirmière et la personne ne repose pas sur des données; elle est de nature humaine. Ce genre de dialogue ne suit pas un scénario préétabli et dans le cadre de cette relation, les infirmières montrent qu'elles s'engagent à être aux côtés des patients pour l'écoute, le respect, le pourvoir et que leur engagement dépendra des vœux des patients (Mitchell, 1999).

Un sixième aspect de l'acquisition des connaissances a été ajouté au modèle conceptuel qui oriente la réflexion sur la pratique que l'on a appelé "méconnaissance" ["unknowing" en anglais]. Ainsi, l'infirmière a conscience qu'elle ne connaît pas et ne peut jamais pleinement connaître la perspective du patient (Munhall, 1993). L'incorporation de cet élément de méconnaissance au modèle revêtait une grande importance pour les infirmières Interlink, car il leur permettait de décrire la composante humaniste de leur pratique comme étant une forme particulière de prestation de soins qui dépasse le domaine strictement médical, et il empêchait de réduire les cas à la simple analyse de leurs éléments empiriques. Les infirmières Interlink reconnaissent que la dispensation de soins de nature humaniste s'accompagne d'un élément de méconnaissance. Ce dernier exige de l'infirmière qu'elle écoute activement son moi et qu'elle soit prête à développer ses connaissances sur l'interaction entre le patient et elle-même afin d'améliorer les soins et le soutien dispensés. La pratique professionnelle exige qu'elle se sente à l'aise avec l'inconnu à la lumière d'un vaste fonds de connaissances et de la compréhension du vécu de la personne aux prises avec le cancer.

Tableau Un: Modèle pour la réflexion structurée (10 ${ }^{\mathrm{e}}$ version)

Modes d'acquisition

du savoir de Carper Questions guidant la réflexion

Dim. esthétique L'art des soins infirmiers

Qu'est-ce que j'essayais d'accomplir? Quels sentiments le patient m'a-t-il décrits?

Pourquoi ai-je réagi comme je l'ai fait?

Quelles en ont été les conséquences?

Dim. personnelle Qu'ai-je ressenti dans cette situation? Par quels facteurs internes, systèmes de croyances ou valeurs ai-je été influencée?

Dim. éthique Dans quelle mesure mes actions correspondaient-elles à mes croyances? Quels principes éthiques étaient en jeu?

Dim. empirique Quelles connaissances auraient dû m'éclairer? Quelle théorie aurait pu m'aider à décrire la situation?

Sur quelles données probantes reposent les interventions utilisées?

Version anglaise reproduite avec la permission de Johns (1995). 
Le tableau deux décrit le modèle de réflexion adopté par les infirmières Interlink lequel incorpore les six modes d'acquisition des connaissances ainsi que les questions dont elles se servent pour orienter leur réflexion.

\section{Utilisation du cadre de réflexion}

Lorsqu'elle utilise le cadre de réflexion, l'infirmière Interlink choisit un patient auquel elle a prodigué des soins dans le cadre de sa pratique et elle se penche sur les soins qu'elle lui a dispensés. Elle peut choisir d'examiner seulement un des éléments du cadre de réflexion ou bien l'ensemble des éléments étant donné que certaines relations infirmière-patient exigent que l'attention porte sur des éléments particuliers. Les infirmières pour qui la démarche d'auto-réflexion est encore une nouveauté préfèrent examiner tous les éléments du cadre.

Lorsqu'elle effectue sa démarche de réflexion, l'infirmière présente un cas particulier à l'équipe infirmière Interlink qu'elle fait suivre d'une réflexion sur les soins prodigués structurée au moyen du cadre. L'infirmière prépare sa réflexion avant de la présenter à l'équipe; elle a donc eu l'occasion de recenser la littérature à la recherche de renseignements supplémentaires qui auraient pu rehausser sa pratique. La réflexion portant sur l'aspect empirique de l'acquisition du savoir infirmier peut amener l'infirmière Interlink à explorer plus à fond un concept ou un construit théorique particulier qui pourrait être utilisé pour améliorer sa pratique. Par exemple, une infirmière Interlink a passé en revue les écrits sur l'adaptation afin d'éclairer sa pratique de manière empirique dans le cas d'une femme éprouvant une détresse importante face à son diagnostic de cancer du sein. Une fois que l'infirmière a terminé sa présentation, l'équipe et une facilitatrice (la Responsable du développement de la pratique) en font une exploration avec l'infirmière et lui fournissent des

Tableau Deux: Cadre de réflexion des infirmières Interlink

Modes

\section{d'acquisition}

Questions guidant la réflexion

Dim. esthétique

L'art des soins infirmiers

Qu'est-ce que j'essayais d'accomplir? Quels sentiments le patient m'a-t-il décrits?

Pourquoi ai-je réagi comme je l'ai fait? Quelles en ont été les conséquences?

Dim. personnelle Qu'ai-je ressenti dans cette situation? Par quels facteurs internes, systèmes de croyances ou valeurs ai-je été influencée?

Dim. éthique Dans quelle mesure mes actions correspondaient-elles à mes croyances? Quels principes éthiques étaient en jeu?

Dim. empirique Quelles connaissances auraient dû m'éclairer?

Quelle théorie aurait pu m'aider à décrire la situation?

Sur quelles données probantes reposent les interventions utilisées?

Dim.

sociopolitique

Où et comment puis-je faire entendre ma voix?

(White, 1995)

Quelle action inductrice de changement est nécessaire pour répondre à ces besoins des patients?

"Méconnaissance" Qu'est-ce qui ne peut s'expliquer dans la (Munhall, 1993) situation du patient et tient tout simplement à la façon d'être de la personne

Adapté avec la permission de Johns (1995). commentaires sur sa pratique en l'orientant vers des connaissances additionnelles ou en lui donnant des conseils pratiques qui auraient pu éclairer sa pratique et pourraient s'avérer utiles dans de futures interactions patient-infirmière. On trouvera ci-dessous un extrait d'une présentation de réflexion sur la pratique.

\section{Réflexion concernant un cas de soins}

L'infirmière rapporte l'histoire de Sally, une femme qui n'a pas loin de 80 ans et qui reste farouchement et fièrement autonome. Cela fait des mois qu'elle se plaint auprès de son médecin de famille de constipation et de plénitude abdominale générale. En fin de ligne, elle se retrouve au service des urgences présentant des saignements vaginaux et une obstruction abdominale. Une opération immédiate révèle un diagnostic de cancer de l'ovaire de stade III. Elle passe ainsi plusieurs semaines à l'hôpital, la plupart d'elles dans l'unité de soins intensifs. Gravement malade, elle a failli mourir. Elle ne se souvient pas clairement de cette période mis à part un commentaire du chirurgien qui lui annonçait qu'il "avait tout enlevé". Sally a hâte de retourner chez elle, mais elle commence à s'inquiéter et à se sentir frustrée quand elle ne retrouve pas sa santé et son énergie d'antan dans la semaine suivant son congé. Moins d'un mois plus tard, elle fait des cauchemars sur son séjour à l'hôpital. Elle refuse l'aide d'un psychiatre. Durant ces jours-là, elle ne veut pas croire qu'on l'a totalement débarrassée de son cancer et elle croit que les docteurs ne lui disent pas tout et qu'ils minimisent ses inquiétudes. Elle concentre ses efforts sur le rétablissement de ses forces d'antan et elle tient à remonter sur son triporteur qui symbolise son autonomie. On lui offre l'aide d'une thérapeute occupationnelle œuvrant en santé mentale communautaire, mais cela ne sert à rien. Durant tout ce temps, elle continue de penser qu'elle souffre encore du cancer car la constipation ne s'est point arrêtée (les radiographies et les examens par IRM s'avèrent négatifs).

\section{La réflexion de l'infirmière}

Dimension esthétique. J'avais de nombreux désirs pour Sally. Je voulais que cessent ses souvenirs pénibles et ses cauchemars effrayants. Je voulais qu'elle ressente moins d'impatience et d'effroi envers sa fatigue, qu'elle se fasse confiance pour l'utilisation de son triporteur et qu'elle soit capable de sortir de son appartement en toute autonomie. Je souhaitais qu'elle reprenne ses activités sociales. C'était ce que Sally m'avait dit qu'elle désirait. J'ai saisi l'angoisse qui marquait sa confidence la plus révélatrice: "Je n'avais jamais pensé que je deviendrais malade et que je mourrais un jour...". Je sentais la profonde relation qui me liait à elle et je désirais alléger son angoisse. Je voulais qu'elle sache que je croyais ce qu'elle disait et que je voulais l'aider. Je pensais que je m'étais ouverte à Sally grâce aux rapports étroits que j'entretenais avec elle et grâce à mon intention de lui venir en aide. Il fallait que je fasse l'effort de ne rien dire, de l'écouter, de débarrasser mes pensées de tout programme. Johns (1995) nous explique que la dimension esthétique de l'acquisition des connaissances est au centre du savoir infirmier et qu'elle est éclairée par les dimensions empirique, personnelle et éthique de la pratique. Dans ma relation avec Sally, la dimension esthétique était la compréhension de l'angoisse qu'elle ressentait après avoir réalisé et admis sa vulnérabilité et sa mortalité.

Dimension personnelle. Plutôt que d'écouter véritablement et d'accompagner Sally dans son angoisse, je me suis mise à essayer de régler ses autres problèmes. Je l'ai abandonnée à son angoisse qui n'a fait qu'augmenter car mes interventions ne donnaient pas de bons résultats. Le but ou "résultat" que j'envisageais - qu'elle retrouve le plus de son autonomie précédente - était aussi le but de Sally. Pourtant, si j'avais bien compris la portée de ce que je savais - que Sally voulait se débarrasser de cette angoisse et qu'elle avait l'habitude de prendre soin d'elle-même - j'aurais abordé Sally en tant qu'être humain ayant bien des questions et des inquiétudes semblables aux miennes à propos de la vulnérabilité et de la mortalité. Nous aurions toutes deux appris quelque chose au sujet de nousmêmes et de l'autre (Parse, 1998). Il se pourrait - ou non - que Sally 
se déplace en triporteur ces temps-ci. Ce qu'elle fait en ce moment ne paraît pas important. Car ce qu'elle fait et ce qu'elle éprouve est moins sous la marque de l'angoisse et plus sous celle de la confiance et de l'espoir (et ce, en dépit de son infirmière et de ses bricolages). Et elle continue de m'aider à apprendre et à découvrir de nouvelles choses dans ma relation avec elle.

Dimension éthique. C'est encore la réflexion qui m'a aidée à comprendre que ce sont les questions sur le savoir éthique qui m'ont permis de réaliser que j'aurais pu accompagner Sally si je l'avais respectée en tant qu'experte sur sa propre vie et si je m'étais ouverte à Sally en tant qu'être humain, et en tant qu'être humain qui ne possède pas toutes les réponses. Mes actions ne correspondaient pas à mes croyances. C'est selon ma propre perspective que je m'efforçais de régler les problèmes pour Sally. Et j'ai mis bien du temps à découvrir la perspective de Sally; en fait, la question du triporteur n'était pas d'importance majeure pour elle. Elle se préoccupait plutôt du fait qu'elle avait été gravement malade, qu'elle en était presque morte et qu'elle n'avait pas encore retrouvé le plein contrôle de sa vie. Une approche éthique des soins aide l'infirmière à fournir une présence empreinte de compréhension aux patients et à entendre leur perspective et les façons dont ils veulent faire face à la maladie qui les frappe.

Dimension empirique. Pour soigner Sally, je dois pouvoir me laisser guider par une perspective théorique plus proche de l'approche humaniste. J'ai examiné les écrits qui portaient sur les réponses infirmières humanistes à des réactions de patients complexes, notamment la "Human - Becoming Theory" de Parse (Parse, 1998). Grâce à Sally, j'ai appris que je pouvais porter attention aux pas en avant et en arrière - aux ambiguïtés et aux contradictions - dans les conversations. La théorie de l'adaptation m'a également aidée à comprendre les réactions traumatiques précoces de Sally à l'annonce du diagnostic telles que ses cauchemars et l'agitation qu'ils créaient en elle.

Dimension sociopolitique. Pour cette situation de pratique, la réflexion ne visait pas la dimension sociopolitique. Cependant, dans d'autres situations, l'infirmière aurait pu s'interroger sur le changement systémique ou sur l'activité de défense des intérêts des patients qui pourraient être nécessaires pour fournir du soutien aux personnes atteintes de cancer et soignées à domicile. La défense des intérêts peut amener l'infirmière à aider d'autres personnes à entendre et à comprendre la perspective du patient.

Méconnaissance. J'ai réalisé que je ne saisissais pas entièrement le vécu de la personne aux prises avec le cancer, et je reconnais que j'ai besoin de m'ouvrir à l'autre et d'entendre son histoire et sa perspective. Il faut que j'écoute activement. Je voulais que Sally sache que les médecins et moi, nous prêtions attention à elle afin qu'elle se croie en bonne santé et qu'elle se concentre sur sa vie. Lorsque j'établissais des buts avec Sally concernant son rétablissement, j'étais assaillie d'une agitation intérieure car je connaissais les probabilités de récurrence de son cancer. Je voulais l'aider à poursuivre sa vie quotidienne. Pour l'aider dans ce cheminement et pour comprendre sa perspective, je lui ai posé un certain nombre de questions d'évaluation du genre: "Qu'est-ce que vous espérez qu'il vous arrive? De quelle aide avez-vous besoin pour cela?" Ces questions engendraient une immense anxiété chez Sally. Elle me demandait de l'aider, de l'orienter et puis elle rejetait les idées avancées. Elle exprimait ses sentiments et ses frustrations, mais à toutes les suggestions proposant des pistes de résolution de problèmes, elle répondait en disant: "Je ne veux pas en parler". Les confidences de Sally entrecoupées de blocages me frustraient. J'ai accordé la priorité à l'écoute et je lui ai posé moins de questions. Toutefois, je n'articulais pas ma frustration - ni même la reconnaissais consciemment. J'ai perdu les priorités de vue. Je voulais être présente auprès de Sally pour elle et selon sa propre perspective et je crois être ouverte à ce genre de pratique. Je crois sincèrement que c'est ainsi que je façonne ma pratique. Pourtant, sans m'en rendre compte, j'écoutais mon propre programme, et c'est ainsi que je me suis mis dans la tête de régler les problèmes reliés à cette situation.

La capacité de l'infirmière à réfléchir sur sa pratique dans une situation comme celle-ci donne des aperçus intimes des approches qu'elle utilisait dans sa pratique et lui permet d'explorer des approches de pratique et de dispensation de soins plus efficaces.

\section{S'adonner à la réflexion sur la pratique}

La connaissance de soi et l'auto-réflexion de l'infirmière représentent les deux éléments les plus difficiles à incorporer aux séances de réflexion sur la pratique qui sont offertes à l'ensemble des membres de l'équipe infirmière Interlink. Et pourtant, ils constituent deux des moyens les plus positifs de rehausser les relations avec les collègues et les patients. La dimension personnelle ou connaissance de soi a trait à l'expérience intérieure d'où émerge la personne entière d'une conscience accrue, et c'est par le truchement de cette connaissance de soi que l'on est à même de comprendre autrui (Chinn et Kramer, 1995).

L'auto-réflexion est un puissant outil qui améliore la compréhension de soi et révèle les raisons du comportement de l'infirmière et les obstacles éventuels à la relation thérapeutique. Les résultats d'une intervention infirmière dépendent nettement de la sorte de personne qu'est la praticienne, et l'infirmière doit avoir conscience de qui elle est afin que les préoccupations d'ordre personnel n'entravent pas l'exploration par le patient de ses propres inquiétudes (Johns, 1995). Certes, le fait de s'impliquer dans une démarche d'auto-réflexion, de révéler la dimension personnelle dans une situation de santé, pourra intimider l'infirmière.

La réflexion sur la pratique donne aux infirmières l'occasion d'articuler les stratégies comportementales qui sous-tendent les interactions et qui les éclairent sur leurs propres schémas de comportement interpersonnel. La perception de ses propres sentiments revêt une importance capitale si on veut comprendre et accepter le patient ou son proche sans préjugés, particulièrement dans un milieu caractérisé par la diversité culturelle. Cependant, il faut reconnaître que la démarche d'auto-réflexion crée un sentiment de vulnérabilité chez l'infirmière car elle abandonne les obstacles liés au rôle professionnel et elle révèle, à ses collègues et à elle-même, sa façon d'exercer. Mais ce n'est que par le truchement d'une démarche d'auto-réflexion que l'on peut résoudre les différences entre les barrières professionnelles et les limites personnelles dans chaque relation infirmière-patient.

\section{Façonner un environnement propice à la réflexion sur la pratique}

La promotion et la mise au point d'un environnement propice à la réflexion font du vécu des infirmières une source d'apprentissage. Les infirmières Interlink sont prêtes à s'impliquer davantage dans l'autoréflexion et dans la réflexion soumise à l'évaluation des pairs. Dans leur cas, l'utilisation de la réflexion sur la pratique au cours des réunions mensuelles de l'équipe infirmière a été un processus lent qui a suivi le rythme du développement des rapports entre les membres de l'équipe. L'orientation du processus a grandement bénéficié de la présence d'une facilitatrice expérimentée qui n'est pas une praticienne Interlink. Le rôle de la facilitatrice est de fournir orientation et rétroaction à l'infirmière individuelle qui utilise le cadre de réflexion sur la pratique et aux membres de l'équipe afin de les encourager à fournir, à leur collègue, des commentaires francs et constructifs. Durant les tout premiers mois, les collègues ont eu tendance à ne donner qu'une rétroaction positive, mais elles sont dorénavant plus à l'aise pour mentionner des interventions qui auraient pu être utiles. L'environnement a été créé graduellement au sein de l'équipe infirmière, et ses membres sont passés d'une réflexion timide sur leur pratique à l'articulation de suggestions d'interventions de rechange. 
Les infirmières Interlink ont éprouvé divers degrés de gêne et d'excitation. Un climat de confiance est d'importance critique si on veut dépasser l'étape du simple soutien accordé aux interventions des collègues pour atteindre une réflexion véritable sur les décisions et les interventions retenues dans la pratique et pour explorer de nouvelles approches possibles de la pratique.

La réflexion sur la pratique exige que l'on remette en question ses croyances anciennes sur les attitudes et les rôles professionnels. Ainsi, les barrières reliées au rôle professionnel peuvent restreindre notre habileté à créer des rapports humanistes et personnels avec les patients. La relation infirmière-patient est un type de relation entre deux personnes, mais elle est plus complexe et elle exige que l'infirmière ait conscience des limites de l'espace personnel si elle veut favoriser la valeur thérapeutique de sa pratique. Par exemple, nous sommes très présentes auprès des patients affligés, il nous arrive même de pleurer avec eux, mais nous ne sommes pas pour autant accessibles durant nos heures hors travail. Lorsqu'elle éprouve des liens affectifs avec les patients - qu'il ne faut pas confondre avec l'engagement affectif - l'infirmière risque d'être peinée par la récurrence de la maladie ou par le décès de patients. En confessant de tels liens affectifs avec les patients à des collègues dans le cadre d'une séance de réflexion sur la pratique, les infirmières peuvent être nombreuses à penser qu'on risque de critiquer leur manque d'efficacité professionnelle.

Il ne faut pas voir dans la réflexion sur la pratique une méthode d'identification des lacunes au niveau de la pratique, mais plutôt un moyen de rehausser notre compréhension de l'art et des connaissances sous-tendant les soins aux patients et à leurs proches et des manières d'améliorer ces soins. Les infirmières chefs et les collègues doivent créer un climat de respect mutuel favorisant un sentiment de confiance et de sécurité afin de minimiser le sentiment de vulnérabilité qui naît obligatoirement de la révélation des façons dont on travaille avec les patients.

\section{Le pouvoir de la réflexion}

L'un des aspects les plus intimidants de la mise en place d'une démarche de réflexion structurée est d'empêcher qu'elle ne devienne un exercice purement intellectuel. Car la réflexion sur la pratique permet éventuellement de révéler et d'articuler les connaissances essentielles sous-tendant la pratique infirmière et d'articuler l'art de la dispensation des soins. On a dit de la réflexion sur la pratique qu'elle était à la fois excitante et terrifiante. Fay (1987) voit dans la réflexion un processus de révélation, d'habilitation et d'émancipation. Par révélation, on entend se comprendre soi-même dans le contexte de la définition et de l'assimilation de la pratique; l'habilitation, c'est avoir le courage et la détermination de prendre les mesures nécessaires pour changer intérieurement; quant à l'émancipation, elle consiste à se libérer de ses anciennes manières d'être et à devenir la personne que l'on doit être pour atteindre les buts fixés en matière de pratique. Pour les infirmières Interlink, la pratique de l'auto-réflexion est une révélation, et elles s'engagent sur la voie de l'habilitation et de l'émancipation.

\section{Conclusion}

L'image de l'infirmière de demain est celle d'une spécialiste du savoir, capable d'articuler les connaissances qui orientent la prise de décision. Les infirmières Interlink trouvent qu'un cadre conceptuel guide leurs pas à mesure qu'elles adoptent la réflexion sur la pratique. Parfois, il leur a été difficile d'explorer en profondeur les situations de patients. Cependant, cette démarche a revêtu une grande importance pour le développement de la qualité de la pratique des infirmières Interlink, et elle a contribué à leur développement individuel et à leur croissance au sein de l'équipe. Il est possible que les infirmières aient besoin d'un certain temps pour perfectionner les compétences liées à la réflexion et d'une longue période de pratique pour intérioriser les signaux issus de la réflexion comme façon de considérer la pratique. Les infirmières à qui on ne donne ni orientation ni encadrement peuvent éprouver des difficultés à atteindre la contemplation et douter de leur capacité à examiner la situation en profondeur, à considérer les raisons de leurs pensées, sentiments et actions. On favorise la réflexion sur la pratique par le biais d'environnements qui offrent à l'infirmière une orientation et un encadrement tandis qu'elle chemine dans cette voie. Les infirmières Interlink s'engagent à utiliser la réflexion en vue de faire progresser constamment la qualité des soins qu'elles dispensent et d'articuler leur rôle en influençant de manière positive le vécu des personnes atteintes de cancer.

\section{Références}

Antrobus, S. (1997). Developing the nurse as a knowledge worker in health - Learning the artistry of practice. Journal of Advanced Nursing, 25, 829-835.

Benner, P. (1984). From novice to expert. Menlo Park, CA: AddisonWesley.

Boykin, A., \& Schoenhofer, S. (1991). Story as link between nursing practice, ontology, epistemology. Image: The Journal of Nursing Scholarship, 23, 245-248.

Bunn, F. (1988). An exploratory study of the role of the Macmillan nurse. Unpublished study, King's College, University of London.

Carper, B.A. (1978). Fundamental patterns of knowing in nursing. Advances in Nursing Science, 1, 13-23.

Chinn, P.L., \& Kramer, M.K. (1995). Theory and nursing a systematic approach. Toronto: Mosby.

Fay, B. (1987). Critical social science. Cambridge, UK: Polity Press. Hagedorn, S. (1995). The politics of caring: The role of activism. Advances in Nursing Science, 17(4), 1-11.

Howell, D. (1998). Parvenir aux profondeurs de l'être: Comprendre et explorer la signification de la maladie. Revue canadienne de soins infirmiers en oncologie (CONJ), 8(1), 17-23.

Johns, C. (1995). Framing learning through reflection within Carper's fundamental ways of knowing in nursing. Journal of Advanced Nursing, 22, 226-234.
Johns, C. (1996). Visualizing and realizing caring in practice through guided reflection. Journal of Advanced Nursing, 24, 1135-1143. Kolb, D.A. (1975). Experiential learning. New Jersey: PrenticeHall.

Lowe, P.B., \& Kerr, C.M. (1998). Learning by reflection: The effect on educational outcomes. Journal of Advanced Nursing, 27, 1030-1033.

Mitchell, G. (1999). Evidence-based practice: Critique and alternative view. Nursing Science Quarterly, 12(1), 30-35.

Munhall, P.L. (1993). "Unknowing": Toward another pattern of knowing in nursing. Nursing Outlook, 41, 125-128.

Parse, R.R. (1998). The Human Becoming school of thought. London, UK: Sage.

Powell, J.H. (1989). The reflective practitioner in nursing. Journal of Advanced Nursing, 14, 824-832.

Schon, D. (1983). The reflective practitioner. New York: Basic Books.

Sheilds, L.E., \& Lindsey, A.E. (1998). Community health promotion nursing practice. Advances in Nursing Science, 20(4), 23-36.

Wansbrough, G. (1996). All aboard for 1997!: CNO embarking on quality assurance program. Communique, 21(1), 14-15.

White, J. (1995). Patterns of knowing: Review, critique, and update. Advances in Nursing Science, 17(4), 73-86. 